\title{
Prevalence of fatigue in adults with congenital heart disease
}

\section{Original Article}

Cite this article: Ternrud L, Hlebowicz J, Sandberg C, Johansson B, and Sparv D (2022) Prevalence of fatigue in adults with congenital heart disease. Cardiology in the Young 32: 1432-1439. doi: 10.1017/S1047951121004297

Received: 11 May 2021

Revised: 28 September 2021

Accepted: 29 September 2021

First published online: 29 October 2021

\section{Keywords:}

Adult congenital heart disease; experience; fatigue; multidimensional fatigue inventory-20; patient-reported outcome

\section{Author for correspondence:}

J. Hlebowicz, Department of Cardiology, Skåne University Hospital, Entrégatan 7, 22185 Lund, Sweden. Tel: +46 46 170000;

Fax: + 4646137658 .

E-mail: joanna.hlebowicz@med.lu.se

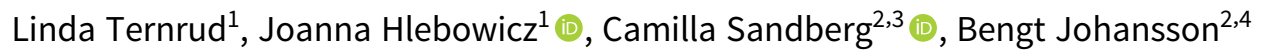 \\ and David Sparv ${ }^{1}$
}

${ }^{1}$ Department of Cardiology, Department of Clinical Science, Skåne University Hospital, Lund University, Lund, Sweden; ${ }^{2}$ Department of Public Health and Clinical Medicine, Umeå University, Umeå, Sweden; ${ }^{3}$ Department of Community Medicine and Rehabilitation, Umeå University, Umeå, Sweden and ${ }^{4}$ Department of Surgery and Perioperative Sciences, Umeå University, Umeå, Sweden

\section{Abstract}

Aims: The aim of this cross-sectional study was to examine the prevalence of the multidimensional phenomenon of fatigue in adults with congenital heart disease. Background: Adults with congenital heart disease are a growing population, and patient-reported outcomes can provide valuable information about the patient's experience of living with CHD. Fatigue is a multidimensional phenomenon that can be described as an overwhelming feeling of exhaustion with a reduced capacity of mental and physical work. Fatigue can be observed clinically in adults with congenital heart disease, but the actual prevalence is unknown. Methods: Fatigue was assessed by the Multidimensional Fatigue Inventory which enables the respondent to report the presence of fatigue according to five dimensions: "general fatigue," "physical fatigue," "mental fatigue," "reduced motivation," and "reduced activity." The questionnaire was sent to 463 patients in Lund and Umeå. Four groups with complex CHD and two groups with moderately complex CHD were included. The reliability (internal consistency) of the Multidimensional Fatigue Inventory was tested for all dimensions and groups of diagnosis. Results: The response rate was $56.6 \%(n=262)$. In patients with complex CHD, 40.0-59.4\% reported severe to very severe general fatigue, and patients with a single ventricle reported the highest prevalence $(59.4 \%)$. Among patients with complex CHD, 29.2-40.0\% reported severe to very severe mental fatigue. The Multidimensional Fatigue Inventory had a high reliability measured with Cronbach's alpha. Conclusions: The study findings show a relatively high prevalence of fatigue in adults with congenital heart disease, and general fatigue was the most prevalent. Further studies are needed regarding fatigue and its causes and consequences in adults with congenital heart disease. Relevance to clinical practice: The Multidimensional Fatigue Inventory proved to be an instrument with high reliability and low internal loss, which suggests that the instrument may be suitable to use as a patient-reported outcome in the care of adults with congenital heart disease, preferably at repeated occasions.
\end{abstract}

Adults with congenital heart disease are a growing population thanks to advances in paediatric cardiology, cardiothoracic surgery, and anaesthesia. ${ }^{1}$ Today, the majority of children born with CHD survive into adulthood. ${ }^{2}$ In Sweden, over $97 \%$ of the children born with CHD reach adulthood. ${ }^{3}$ Despite the advances in surgical treatment and medical care, most of the patients cannot be considered as cured. The majority of patients need regular follow-up to monitor and detect residual problems such as arrhythmias, heart failure, and need of re-interventions. ${ }^{4} \mathrm{CHD}$ is therefore increasingly considered as a lifetime disease with specific trajectories. ${ }^{5}$

Heart failure is the leading cause of death in patients with $\mathrm{CHD} .{ }^{6}$ Furthermore, patients with a single ventricle circulation and patients with a systemic right ventricle (congenitally corrected transposition of the great arteries and transposition of the great arteries operated on with the Mustard/Senning technique) are at higher risk of hospitalisation for heart failure compared to patients with more simple heart lesions. ${ }^{7}$ The prevalence of heart failure in patients with a single ventricle is approximately $40 \%$, in patients who had a Mustard/Senning procedure $22 \%$ and in patients with congenitally corrected transposition of the great arteries the prevalence is $32 \% .{ }^{8}$

Fatigue is a common symptom in patients with heart failure ${ }^{9}$ and can be described as an overwhelming and sustained feeling of exhaustion with a reduced capacity for mental and physical work. Furthermore, fatigue is a complex, multidimensional, and subjective phenomenon. ${ }^{10}$ The experience of fatigue in patients with heart failure is described as internal, dynamic and requires planning and adaptation of their daily lives. ${ }^{11}$ Fatigue is a symptom that can be observed clinically in adults with congenital heart disease but the prevalence of fatigue as a multidimensional phenomenon and the impact on the patient's daily life has been poorly studied.

The patient's experience of their disease is a priority in the research of adults with congenital heart disease to better understand better the long-term impact of the disease on the patient's 
daily life. ${ }^{12}$ A patient-reported outcome is any report of the patient's health condition that comes directly from the patient without any interpretation of any healthcare provider and can be used to understand the patient's subjective experience of a disease. ${ }^{13}$ Fatigue as a multidimensional phenomenon can be assessed by a patient-reported outcome called the Multidimensional Fatigue Inventory ${ }^{14}$ and has previously been used in other patient groups to assess fatigue, for example in patients with heart failure. ${ }^{9}$

The number of studies using a patient-reported outcome in the field of adults with congenital heart disease is growing, and various aspects have been studied, such as quality of life, psychological functioning, health behaviours and perceived health. ${ }^{15,16,17}$ Several aspects of living with congenital heart disease have not yet been covered, and the primary aim of this study was to study the prevalence of the multidimensional phenomenon of fatigue in adults with congenital heart disease using the Multidimensional Fatigue Inventory. A second aim was to test the reliability of the Multidimensional Fatigue Inventory in this population.

\section{Methods}

\section{Study design and participants}

This study was performed with a cross-sectional design using a 20-item self-report questionnaire called the Multidimensional Fatigue Inventory. ${ }^{14}$ The questionnaire was sent by postal mail to 463 adult (age $>18$ years) patients with a planned follow-up at Skåne University hospital in Lund, Sweden $(n=307)$ or Umeå University Hospital, Sweden $(n=156)$. The patients were identified by the Swedish Registry of Congenital Heart Disease (SWEDCON) which is a national registry that consists of four parts: foetal heart diagnostics, paediatric cardiology, adults with congenital heart disease, and congenital heart surgery. The purpose of the register is to provide information about the natural course and treatments for different congenital heart conditions.

Based on basic anatomy and the chosen treatment method, heart defects can be classified as simple (I), moderately complex (II) or complex (III). ${ }^{18}$ The included diagnosis groups were coarctatio aortae (II) $(n=185)$, tetralogy of Fallot (II) $(n=112)$, transposition of the great arteries operated with atrial switch (Mustard/Senning) (III) $(\mathrm{n}=41)$, or arterial switch (III) $(\mathrm{n}=47)$, congenitally corrected transposition of the great arteries (III) $(\mathrm{n}=23)$ and patients with a single ventricle circulation (Fontan/ Total cavopulmonary connection) (III) $(n=55)$. Median age of the included patients was 32.5 years (IQR 17.3); 63.9\% $(\mathrm{n}=296)$ were men, and $36.1 \%(n=167)$ were women. The sample was consecutive but patients with an inability to understand the Swedish language and patients with cognitive dysfunction were not included. The participants were reminded after 3-4 weeks by phone, and those who were not reached at that time received a new questionnaire by postal mail.

All participants gave their written informed consent. The study was approved by the Regional Ethics Review Board in Umeå, Sweden (dnr 2018-238-32M). The investigation conforms to the principles outlined in the Declaration of Helsinki. ${ }^{19}$

\section{Multidimensional fatigue inventory}

The prevalence of fatigue was assessed using the Multidimensional Fatigue Inventory that enables the respondent to report the presence of fatigue according to five dimensions. The five dimensions are "general fatigue," "physical fatigue," "mental fatigue," "reduced motivation" and "reduced activity." General fatigue includes general statements of fatigue, such as "I feel tired" or "I am rested." Physical fatigue describes fatigue connected to physical functions and mental fatigue refers to mental challenges, such as difficulties in maintaining concentration. Reduced activity and reduced motivation can be described as consequences of fatigue. ${ }^{14}$

The questionnaire refers to the experience of fatigue during the last few days, and the questions were answered on a five-degree Likert scale. Each dimension consists of four questions, and the points from each question were added up with a total score ranging between 4 and 20. Higher scores indicate higher levels of fatigue. ${ }^{14}$ The scores can be categorised as none (1-4), mild (5-8), moderate $(9-12)$, severe (13-16), and very severe fatigue $(17-20){ }^{9}$

The reliability (internal consistency) of the subscales in the Multidimensional Fatigue Inventory has been tested with Cronbach's alpha in different populations. ${ }^{9,20,21}$ The creators of the questionnaire obtained values between 0.76 and 0.93 for all dimensions in five populations except in the dimension of reduced motivation where lower values were obtained (0.66 and 0.57$){ }^{9}$ In the Swedish version, one study obtained values between 0.75 and 0.94 for all dimensions ${ }^{20}$ and another study with four populations obtained values between 0.72 and 0.94 except for one lower value in the dimension "reduced motivation" (0.67) and one lower value in the dimension of 'mental fatigue' $(0.68) .{ }^{21}$

The convergent validity of the Multidimensional Fatigue Inventory has been tested. ${ }^{14,21}$ The correlation between Multidimensional Fatigue Inventory and Visual Analogue Scale ranged between 0.23 and $0.77(\mathrm{p}<0.01)$, and general fatigue had the highest correlation (0.77). ${ }^{14}$ The correlation between the Multidimensional Fatigue Inventory and Category Ratio instrument (CR-10) ranged between 0.37 and $0.74(\mathrm{p} \leq 0.001)$, and general fatigue had the highest correlation $(0.74) .{ }^{21}$

\section{Statistical analysis}

Descriptive data are presented as frequencies with percent and median with interquartile range. Comparison of age between participants and non-participant was analysed using the Mann-Whitney $U$-test. Comparison of sex distribution between participants and non-participants was analysed with the $\chi^{2}$-test. Data from the Multidimensional Fatigue Inventory were interpreted as ordinal data and presented as median values with IQR. Comparison between diagnosis groups was analysed using the Kruskal-Wallis H-test. The reliability (internal consistency) in terms of Cronbach's alpha was calculated for each diagnosis group and dimension of fatigue. Values between 0.70 and 0.89 indicate good reliability and values above 0.90 high reliability. ${ }^{22}$ The internal loss (i.e. missing data) was six (2\%) incomplete questionnaires out of 262. Due to low number of missing data, no specific statistical measures were taken.

Statistical analyses were performed using the Statistical Package for Social Sciences version 26 (SPSS, IBM corp., Armonk, NY, US). The null hypothesis was rejected for p-values $<0.05$.

\section{Results}

The Multidimensional Fatigue Inventory was sent to 463 patients. The response rate was $56.6 \%(\mathrm{n}=262)$ and varied between groups with the highest response rate in the congenitally corrected transposition of the great arteries group $(73.9 \%, n=17)$, Table 1 . There was a difference in age between the participants and nonparticipants, 35.6 years (IQR 21.9) vs. 30.0 years (IQR 11.8), $(\mathrm{p}<0.001)$. There was also a difference in sex distribution between 
Table 1. Included patients and response rate

\begin{tabular}{|c|c|c|}
\hline Diagnosis (classification) & $\mathrm{n}$ & Response rate, $\mathrm{n}(\%)$ \\
\hline Coarctatio aortae (II) & 185 & $97(52.4)$ \\
\hline Tetralogy of Fallot (II) & 112 & $67(59.8)$ \\
\hline Mustard/Senning (III) & 41 & $24(58.5)$ \\
\hline Transposition of the great arteries operated with arterial switch (III) & 47 & $25(53.2)$ \\
\hline Congenitally corrected transposition of the great arteries (III) & 23 & $17(73.9)$ \\
\hline Fontan/Total cavopulmonary connection (III) & 55 & $32(58.2)$ \\
\hline Total sample & 463 & $262(56.6)$ \\
\hline
\end{tabular}

II: moderately complex CHD; III: complex CHD.

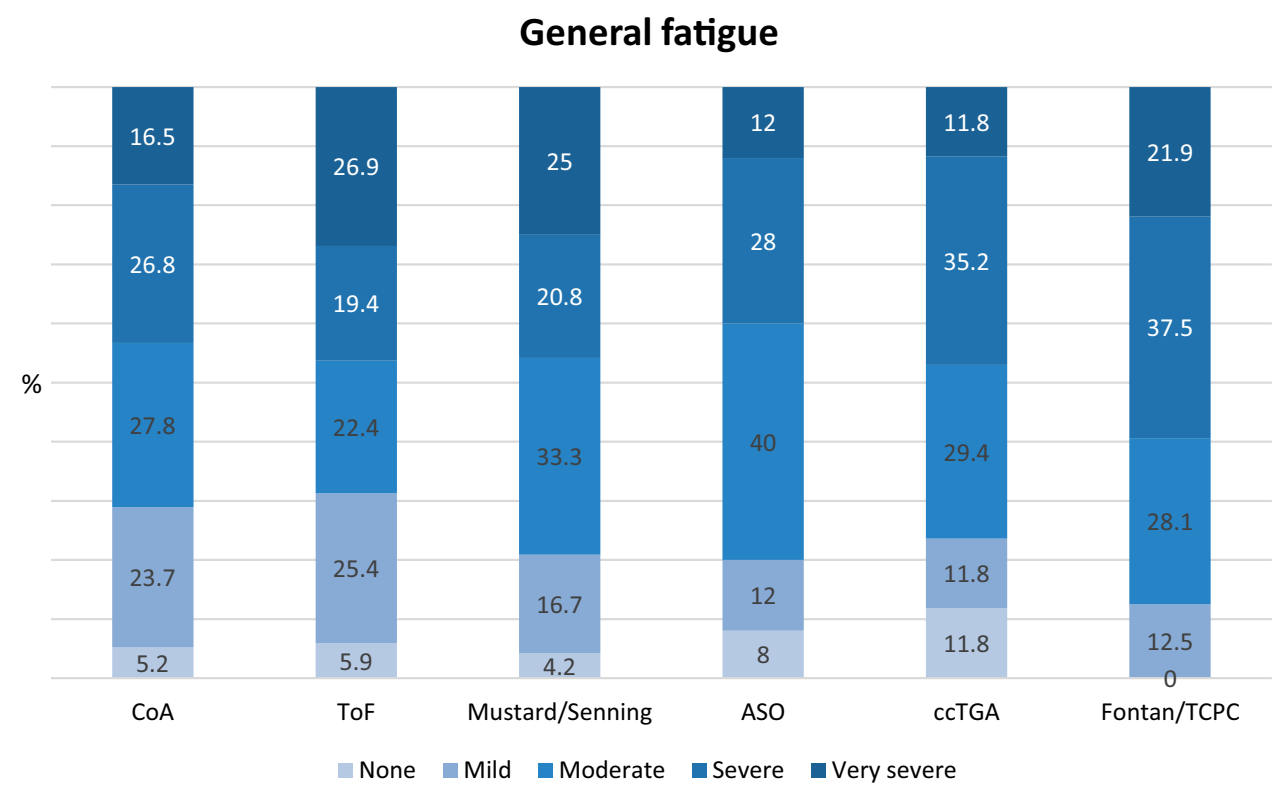

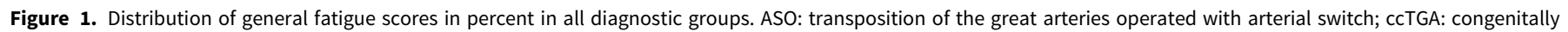
corrected transposition of the great arteries; CoA: coarctatio aortae; TCPC: total cavopulmonary connection; ToF: tetralogy of Fallot.

the participants $(59.5 \%$ men vs. $40.5 \%$ women $)$ and the nonparticipants $(69.7 \%$ men vs. $30.3 \%$ women $),(\mathrm{p}<0.05)$.

\section{General fatigue}

In the single ventricle circulation group, $59.4 \%$ stated that they experienced severe to very severe general fatigue. All of the patients stated that they experienced some degree of general fatigue. In the congenitally corrected transposition of the great arteries group, $47.0 \%$ stated that they experienced severe to very severe general fatigue. The patients with arterial switch had the lowest prevalence of severe to very severe general fatigue, $40.0 \%$, Figure 1 .

\section{Physical fatigue}

In the Mustard/Senning group, $37.5 \%$ experienced severe to very severe physical fatigue and $36.0 \%$ in the arterial switch group experienced severe to very severe physical fatigue. Patients with coarctatio aortae had the lowest prevalence of severe to very severe physical fatigue $(28.9 \%)$, but the variation between the groups was not large, Figure 2.

\section{Mental fatigue}

The two groups with the highest prevalence of severe to very severe mental fatigue were the arterial switch group (40.0\%) and the single ventricle circulation group (37.6\%). None of the single ventricle circulation patients stated that they did not experience any mental fatigue at all. Coarctatio aortae was the group with the lowest prevalence of severe to very severe mental fatigue (19.6\%), Figure 3.

\section{Reduced motivation}

The group of patients with congenitally corrected transposition of the great arteries had the highest prevalence of severe to very severe reduced motivation $(17.7 \%)$. In the other five diagnosis groups, the prevalence was $11.9 \%$ as the most, of severe to very severe reduced motivation, Figure 4.

\section{Reduced activity}

In the dimension of reduced activity, $52.0 \%$ in the arterial switch group experienced severe to very severe reduced activity. The Mustard/Senning group reported that $41.7 \%$ experienced severe to very severe reduced activity. The tetralogy of Fallot group 
Physical fatigue

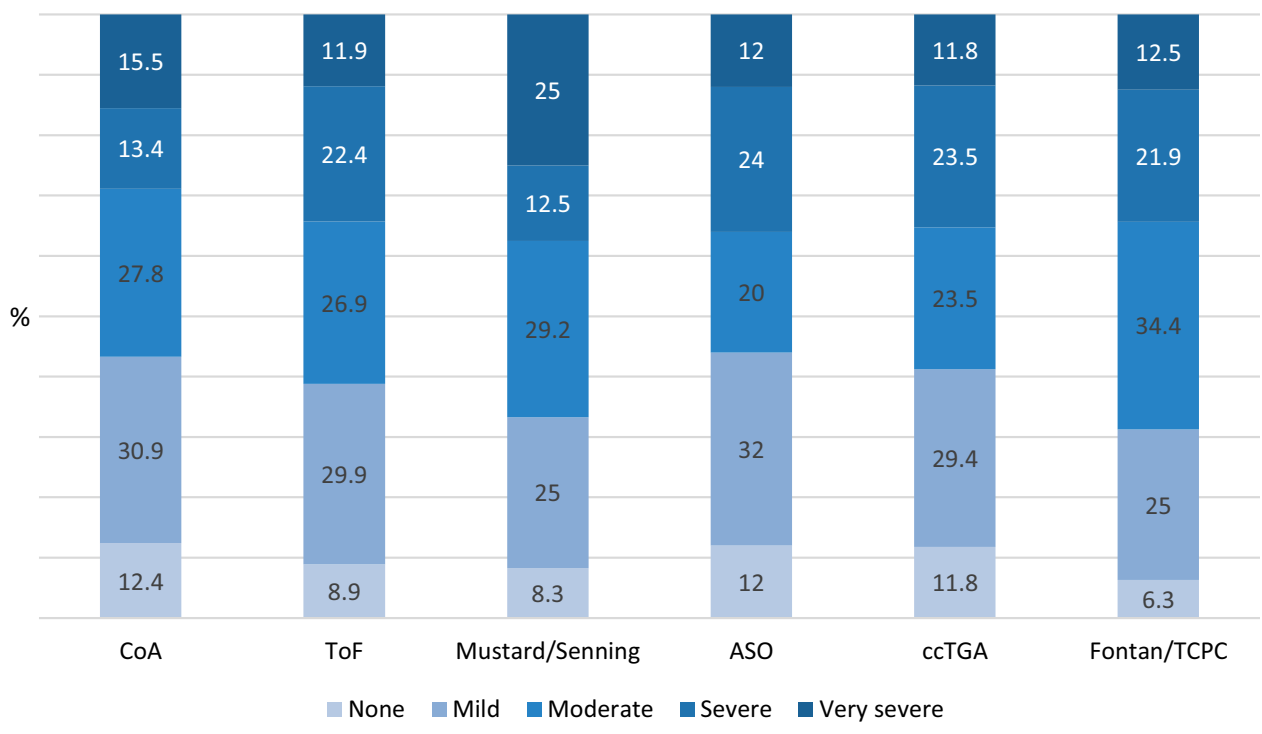

Figure 2. Distribution of physical fatigue scores in percent in all diagnostic groups. ASO: transposition of the great arteries operated with arterial switch; ccTGA: congenitally corrected transposition of the great arteries; COA: coarctatio aortae; TCPC: total cavopulmonary connection; ToF: tetralogy of Fallot.

\section{Mental fatigue}

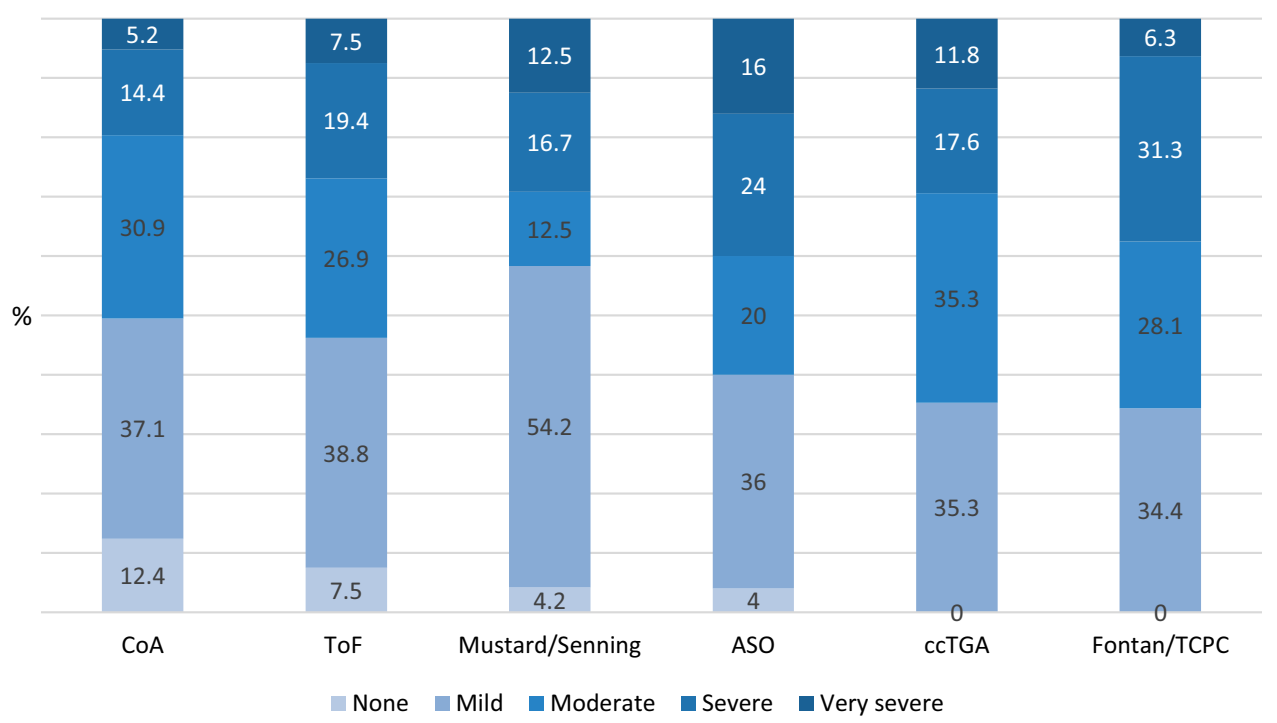

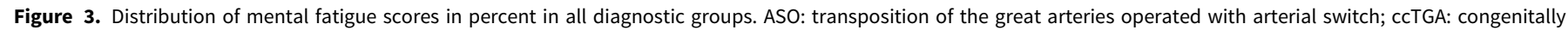
corrected transposition of the great arteries; CoA: coarctatio aortae; TCPC: total cavopulmonary connection; ToF: tetralogy of Fallot.

reported the lowest prevalence with $23.9 \%$ experiencing severe to very severe reduced activity, Figure 5.

\section{Median scores}

The median scores showed that two diagnosis groups reported median scores that fell within the category of severe fatigue. The single ventricle circulation group had a median score of 13 in the dimension of "general fatigue" and the arterial switch group had a median score of 13 in the dimension of "reduced activity." There was no difference between the groups in any of the dimensions, Table 2.

\section{Reliability test}

The reliability (internal consistency) calculated with Cronbach's alpha generated values between 0.74 and 0.93 for all patient groups and dimensions except for the single ventricle circulation group in the dimension of "reduced motivation" (0.49), Table 3.

\section{Discussion}

This cross-sectional study on prevalence of self-reported fatigue in adults with congenital heart disease assessed with Multidimensional Fatigue Inventory showed that "general fatigue" 
Table 2. Fatigue score for each dimension and diagnostic group with test of significance

\begin{tabular}{|c|c|c|c|c|c|c|c|}
\hline & $\mathrm{COA}$ & ToF & Mustard/Senning & ASO & cCTGA & Fontan/TCPC & $p$-value \\
\hline General fatigue & $11(8)$ & $12(9)$ & $11(8)$ & $11(7)$ & $12(6)$ & $13(6)$ & 0.747 \\
\hline Physical fatigue & $10(8)$ & $10(9)$ & $10(9)$ & $9(7)$ & $10(7)$ & $11.5(7)$ & 0.732 \\
\hline Mental fatigue & $9(6)$ & $9(7)$ & $8(8)$ & $9(8)$ & $10(7)$ & $10(7)$ & 0.357 \\
\hline Reduced motivation & $7(5)$ & $7(5)$ & $7(5)$ & $7(6)$ & $7(5)$ & $8(4)$ & 0.955 \\
\hline Reduced activity & $9(7)$ & $9(5)$ & $9(12)$ & $13(7)$ & $10(6)$ & $11(5)$ & 0.267 \\
\hline
\end{tabular}

Fatigue score is presented as median (interquartile range).

The score is categorised as none (4), mild (5-8), moderately (9-12), severe (13-16), and very severe (17-20). Difference between groups is tested with Kruskal-Wallis H-test.

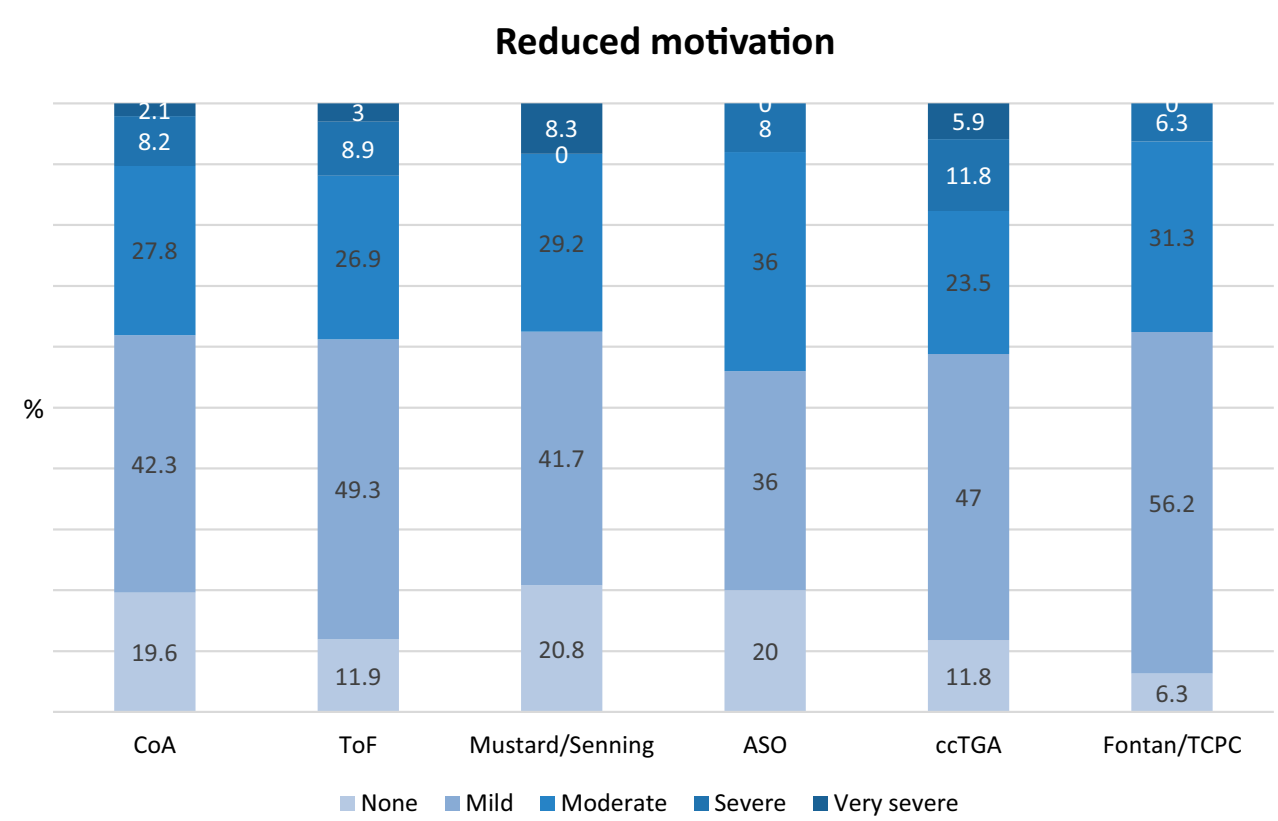

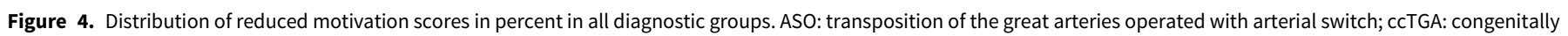
corrected transposition of the great arteries; CoA: coarctatio aortae; TCPC: total cavopulmonary connection; ToF: tetralogy of Fallot.

\section{Reduced activity}

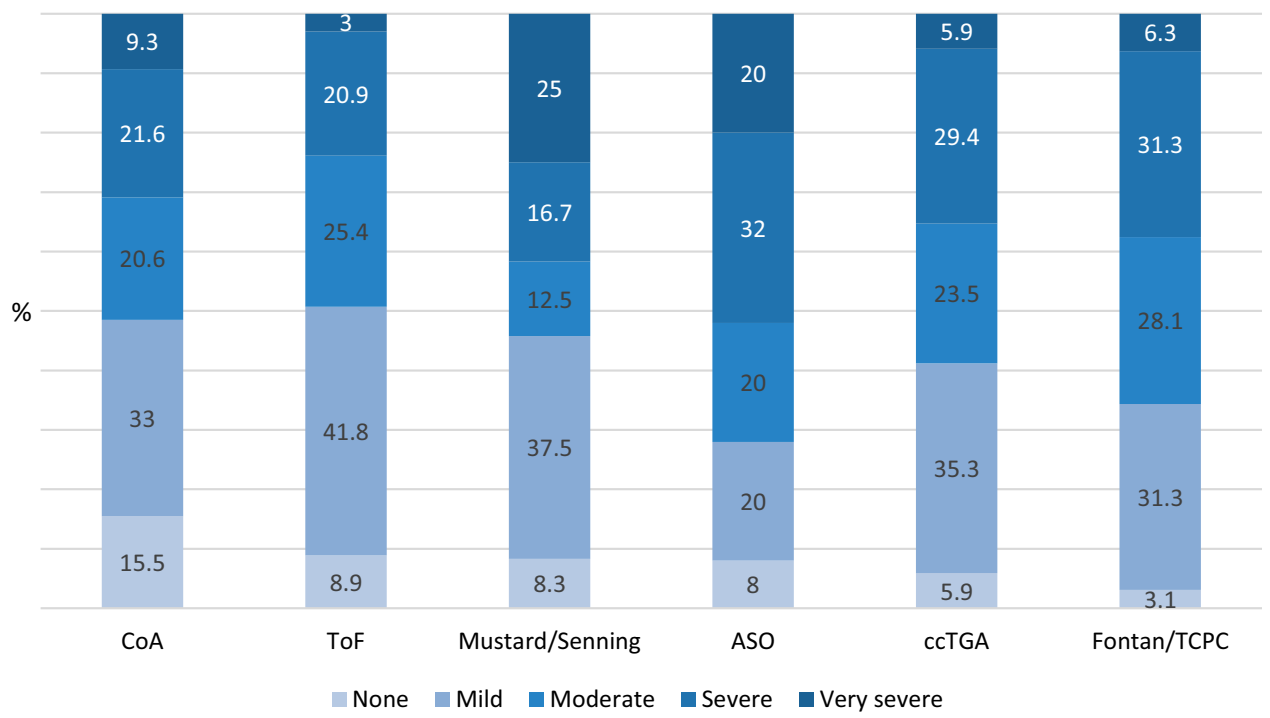

Figure 5. Distribution of reduced activity scores in percent in all diagnostic groups. ASO: transposition of the great arteries operated with arterial switch; ccTGA: congenitally corrected transposition of the great arteries; CoA: coarctatio aortae; TCPC: total cavopulmonary connection; ToF: tetralogy of Fallot. 
Table 3. Internal consistency measured with Cronbach's alpha

\begin{tabular}{|c|c|c|c|c|c|c|}
\hline & $\mathrm{COA}$ & ToF & Mustard/Senning & ASO & CCTGA & Single ventricle \\
\hline General fatigue & 0.90 & 0.87 & 0.87 & 0.82 & 0.86 & 0.83 \\
\hline Physical fatigue & 0.93 & 0.89 & 0.88 & 0.92 & 0.91 & 0.90 \\
\hline Mental fatigue & 0.86 & 0.83 & 0.87 & 0.91 & 0.88 & 0.88 \\
\hline Reduced motivation & 0.75 & 0.80 & 0.80 & 0.74 & 0.84 & 0.49 \\
\hline Reduced activity & 0.92 & 0.84 & 0.93 & 0.88 & 0.88 & 0.83 \\
\hline
\end{tabular}

was the most prevalent dimension of fatigue for these patients, especially for the single ventricle circulation group. The dimensions of "physical fatigue," "mental fatigue," and "reduced activity" were also prevalent to different extents for the patients. Reduced motivation was not as prevalent as the other dimensions which correspond to our clinical experience and observations of these patients. The Multidimensional Fatigue Inventory showed a high reliability measured with Cronbach's alpha.

In our study, more than half of the single ventricle circulation patients experienced severe to very severe general fatigue. The congenitally corrected transposition of the great arteries group and the Mustard/Senning group also reported high values of severe to very severe general fatigue and about one-third severe to very severe physical fatigue. In these groups, the prevalence of heart failure is reported as high. ${ }^{7}$ The study on patients with heart failure reported that the dimension of "physical fatigue" was the most prevalent, with about $80 \%$ of the patients suffering from severe to very severe physical fatigue. ${ }^{9}$ The patients in our study did not express the same pattern of fatigue as the patients with heart failure. Patients with heart failure experienced the dimension of "physical fatigue" as the most prevalent and adults with CHD experienced "general fatigue" as the most prevalent. The difference in self-reported fatigue between patients with heart failure and adults with CHD may be an indication that they suffer from another kind of fatigue which is most easily expressed in the dimension of "general fatigue."

The arterial switch group reported the highest prevalence of severe to very severe mental fatigue. All of the patient groups with complex CHD seemed to experience more severe to very severe mental fatigue compared to the patients with moderately complex CHD. Children born with CHD are at higher risk of developmental disorders and delays, especially children with complex and moderately complex heart defects. Changes in the flow and oxygenation of the blood, both when the child is in the uterus and after birth, may affect brain development. In addition, during infancy, the child undergoes surgery, catheter interventions, and medical treatments at a stage when the brain is developing and sensitive. Children who are operated upon with an arterial switch have their surgery very early in life and have a high risk of developing developmental disorders, with regard to cognitive and executive functions. ${ }^{23}$ This might explain the high prevalence of severe to very severe mental fatigue in this patient group in our study.

More than half of the arterial switch group reported severe to very severe reduced activity, and this was the group with the highest prevalence within that dimension. "Reduced activity" and "reduced motivation" could be seen as consequences of general, physical, and mental fatigue. ${ }^{14}$ If that is the case, our study shows that the most prominent effect on the adults with congenital heart disease patients' lives is "reduced activity" and not "reduced motivation." This corresponds to our clinical observations of the patients, in that they do not often express a lack of motivation but instead a lack of energy.

The severity of fatigue can be categorised as none, mild, moderate, severe, and very severe. ${ }^{9}$ It is not known to what extent each category impacts upon the patient's daily life. If the daily life is affected at the level of moderate fatigue, then the majority of the single ventricle circulation patients suffer from general and mental fatigue to such an extent that it impacts upon their daily lives. It is of importance to understand to what extent the daily life is affected in each category to be able to know when the patient might need help to manage their situation. We hope to address this question in future studies.

The reasons for fatigue in adult patients with CHD might be multifactorial. All dimensions of fatigue could hypothetically be linked to several factors, for example the degree of physical activity, muscle function, age, and heart failure. Fatigue measured with the Multidimensional Fatigue Inventory is described in the general population in Sweden. Higher levels of activity were associated with lower levels of fatigue for all subscales except mental fatigue. ${ }^{24}$ A recent study showed that adults with CHD are as physically active as healthy controls, but half of the study participants did not reach the WHO recommendations for physical activity. ${ }^{25}$ However, it is difficult to know what causes what, a less physically active person may be less active due to a high degree of fatigue and a lack of physical activity can cause fatigue. Furthermore, adult patients with complex CHD showed impaired muscle function in comparison to healthy controls ${ }^{26}$ which might explain the presence of physical fatigue. Individualised support to increased physical activity might support adult patients with CHD that experience fatigue and guidance from a physiotherapist can therefore be valuable. In the general population in Sweden, fatigue scores were generally lower with higher age but other studies have reported the opposite, ${ }^{24}$ which makes it difficult to draw any conclusions about the low median age in our study population and the prevalence of fatigue.

In a qualitative study of the experience of living with a single ventricle, most of the respondents had learned to live with their physical limitations and accepted them as a part of life. ${ }^{27}$ Adults with congenital heart disease have nothing else to compare with since they are born with their condition. Healthcare personnel might observe that the patient's level of fatigue is not within the normal range, but the patient feels like they are in their normal state. It is possible that adult patients with $\mathrm{CHD}$ only report having fatigue when they are in a state of deterioration which might imply that repeated measurements with the Multidimensional Fatigue Inventory could be valuable to detect signs of deterioration.

Different symptoms are registered in SWEDCON, such as being tired, which should be separated from the phenomenon of fatigue. Fatigue is a complex, multidimensional, and subjective phenomenon that does not disappear after a good night's rest 
(tiredness). ${ }^{10}$ The symptoms registered in SWEDCON, however, are mainly registered according to the caregiver's assessment. When symptoms are not reported by the patient, asked for or obvious during the visit, the patient could be considered asymptomatic in SWEDCON. ${ }^{28}$ Fatigue is described as an inner experience that others cannot see ${ }^{10}$ and adults with CHD might avoid informing about symptoms and discomfort due to fear of deterioration and further examinations or procedures. The caregiver might also interpret a patient's symptoms based on their own starting point and reduce or enlarge symptoms depending on their own experiences or previous contacts with other patients. Thus, there may be several reasons to highlight the importance of patient-reported outcomes and the patient's subjective experience and not the caregiver's interpretation of the patient's symptoms.

\section{Limitations}

The Multidimensional Fatigue Inventory refers to the experience of fatigue in the last few days. The answers could have been affected by external factors such as infection, depression, or lack of sleep which is a threat to the internal validity and a limitation of the study. This might be an additional reason to use the Multidimensional Fatigue Inventory under repeated occasions.

It is impossible to rule out that fatigue itself was not responsible for non-response in some of the non-responder groups. However, the congenitally corrected transposition of the great arteries group and the Mustard/Senning group reported high values of severe to very severe general fatigue and also had the highest response rate, $73.9 \%$ and $58.5 \%$, respectively.

Reasons for the lower value of reliability in the dimension of "reduced motivation" in our study could be due to missing values but the fact that other studies also obtained lower values in that dimension might imply that there is a problem with the reliability of the subscale for 'reduced motivation'.

Furthermore, in this study there were no healthy controls or other control group which is also a limitation. In the general Swedish population, age 25-74 years, the mean general fatigue has been reported 9.7 for men and 10.8 for women, mean physical fatigue 9.0 for men and 9.7 for women, reduced activity 9.0 for men and 9.3 for women and mental fatigue 8.4 for men and 8.7 for women. ${ }^{24}$ The fatigue scores in this study were calculated in terms of median instead of mean which makes the values difficult to compare with the values of the general population.

The prevalence of heart failure in our study is not known, which is a limitation, but the prevalence of general fatigue and physical fatigue were still high. This might indicate that heart failure may not be the only reason for fatigue in adults with congenital heart disease.

\section{Conclusion}

In summary, this study demonstrates to what extent adult patients with certain moderately complex and complex heart defects experience different dimensions of fatigue. Adults with congenital heart disease experience of fatigue do not appear to follow the same pattern as for patients with heart failure, suggesting that there could be other explanations for fatigue in these patients. The Multidimensional Fatigue Inventory proved to be an instrument with high reliability and low internal loss, which suggests that the instrument may be suitable to use as a patient-reported outcome in the care of and in studies of adults with congenital heart disease, preferably at repeated occasions.

\section{Relevance to clinical practice}

- Adults with congenital heart disease may experience different dimensions of fatigue.

- The Multidimensional Fatigue Inventory can be used as a patient-reported outcome in adults with congenital heart disease.

- The Multidimensional Fatigue Inventory should preferably be used repeatedly.

Acknowledgements. The authors wish to express their thanks to Caroline Löfvenmark at Sophiahemmet Högskola in Stockholm, Sweden, for valuable advice and guidance.

Financial support. The study was funded by Heart-Lung Foundation, Regional research support and Skåne University Hospital foundations and donations.

\section{Conflicts of interest. None.}

\section{References}

1. Van der Linde D, Konings EE, Slager MA, et al. Birth prevalence of congenital heart disease worldwide: a systematic review and meta-analysis. J Am Coll Cardiol 2011; 58: 2241-2247.

2. Moons P, Bovijn L, Budts W, et al. Temporal trends in survival to adulthood among patients born with congenital heart disease from 1970 to 1992 in Belgium. Circulation 2010; 122: 2264-2272.

3. Mandalenakis Z, Giang KW, Eriksson P, et al. Survival in children with congenital heart disease: Have we reached a peak at 97\%? J Am Heart Assoc 2020; 9: 1-8.

4. Warnes CA. The adult with congenital heart disease. Born to be bad? J Am Coll Cardiol 2005; 46: 1-8.

5. Marelli A. Trajectories of care in congenital heart disease - the long arm of disease in the womb. J Intern Med 2020; 288: 390-399.

6. Zomer AC, Vaartjes I, Uiterwaal C, et al. Circumstances of death in adult congenital heart disease. Int J Cardiol 2012; 154: 168-172.

7. Zomer AC, Vaartjes I, vad der Elde ET, et al. Heart failure admissions in adults with congenital heart disease; risk factors and prognosis. Int J Cardiol 2013; 168: 2487-2493.

8. Piran S, Veldtman G, Siu S, et al. Heart failure and ventricular dysfunction in patients with single or systemic right ventricles. Circulation 2002; 105: 1189-1194.

9. Falk K, Swedberg K, Gaston-Johansson F, et al. Fatigue is a prevalent and severe symptom associated with uncertainty and sense of coherence in patients with chronic heart failure. Eur J Cardiovasc Nurs 2007; 6: 99-104.

10. Tiesinga LJ, Dassen TWN, Halfens RJG. Fatigue: a summary of the definitions, dimensions and indicators. Nurs Diagn 1996; 7: 51-62.

11. Jones J, McDermott CM, Nowels CT, et al. The experience of fatigue as a distressing symptom of heart failure. Heart Lung 2012; 41: 484-491.

12. Goossens E, Fleck D, Cannobio MM, et al. Development of an international research agenda for adult congenital heart disease nursing. Eur J Cardiovasc Nurs 2012; 12: 7-16.

13. Food and Drug Administration (FDA). Guidance for industry. Patient-reported outcome measures. Use in medical product development to support medical claims, 2009. https://www.fda.gov/media/77832/download

14. Smets EMA, Garssen B, Bonke B, et al. The multidimensional fatigue inventory (MFI): psychometric qualities of an instrument to assess fatigue. J Psychosom Res 1995; 39: 315-325.

15. Apers S, Kovacs AH, Luyckx K, et al. Assessment of patterns of patientreported outcomes in adults with congenital heart disease - International study (APPROACH-IS): rationale, design and methods. Int J Cardiol 2015; 179: 334-342.

16. Apers S, Kovacs AH, Luyckx K, et al. Quality of life of adults with congenital heart disease in 15 countries: evaluating country-specific characteristics. J Am Coll Cardiol 2016; 67: 2237-2245. 
17. Moons P, Luyckx K, Thomet C, et al. Physical functioning, mental health, and quality of life in different congenital heart defects: comparative analysis in 3538 patients from 15 countries. Can J Cardiol 2021; 32: 215-223.

18. Stout KK, Daniels CJ, Aboulhosn JA, et al. 2018 AHA/ACC guideline for the management of adults with congenital heart disease: executive summary. J Am Coll Cardiol 2019; 73: 81-192.

19. Rickham PP. Human experimentation: code of ethics of the World Medical Association (declaration of Helsinki). Br Med J 1964; 2: 177.

20. Fürst CJ, Åhsberg E. Dimensions of fatigue during radiotherapy. An application of the Multidimensional Fatigue Inventory. Support Care Cancer 2001; 9: 355-360.

21. Lundh Hagelin C, Wengström Y, Runesdotter S, et al. The psychometric properties of the Swedish Multidimensional Fatigue Inventory MFI-20 in four different populations. Acta Oncol 2007; 46: 97-104.

22. Olsson H, Sörensen S. Forskningsprocessen. 3rd edn. Liber, Stockholm, 2011, 124

23. Marino BS, Lipkin PH, Newburger JW, et al. Neurodevelopmental outcomes in children with congenital heart disease: evaluation and management. Circulation 2012; 126: 1143-1172.
24. Engberg I, Segerstedt J, Waller G, et al. Fatigue in the general populationassociations to age, sex, socioeconomic status, physical activity, sitting time and self-rated health: the northern Sweden Monica study 2014. BMC Public Health 2017; 17: 1-9.

25. Sandberg C, Pomeroy J, Thilén U, et al. Habital physical activity in adults with congenital heart disease compared with age- and sex-matched controls. Can J Cardiol 2016; 32: 547-553.

26. Sandberg C, Thilén U, Wadell $\mathrm{K}$, et al. Adults with complex congenital heart disease have impaired skeletal muscle function and reduced confidence in performing exercise training. Eur J Prev Cardiol 2015; 22: 1523-1530.

27. Overgaard D, King C, Christensen RF, et al. Living with half a heart experiences of young adults with single ventricle physiology: a qualitative study. J Cardiovasc Nurs 2013; 28: 187-196.

28. Berghammer M, Karlsson K, Ekman I, et al. Self-reported health status (EQ-5D) in adults with congenital heart disease. Int J Cardiol 2011; 165: 537-543. 